\title{
The axial anomaly of Ginsparg-Wilson fermion
}

\author{
Ting-Wai Chiu ${ }^{1}$ \\ Department of Physics, National Taiwan University, Taipei 106, Taiwan, ROC \\ Received 22 October 1998; revised 20 November 1998 \\ Editor: M. Cvetič
}

\begin{abstract}
The axial anomaly of Ginsparg-Wilson fermion operator $D$ is discussed in general for the operator $R$ which enters the chiral symmetry breaking part in the Ginsparg-Wilson relation. The axial anomaly and the index of $D$ as well as the exact realization of the Atiyah-Singer index theorem on the lattice are determined solely by the topological characteristics of the chirally symmetric operator $D_{c}$ in the chiral limit $R \rightarrow 0$. C 1999 Published by Elsevier Science B.V. All rights reserved.
\end{abstract}

PACS: 11.15.Ha; 11.30.Rd; 11.30.Fs

The Ginsparg-Wilson (GW) relation [1] has been playing an important role in recent theoretical developments of the chiral symmetry on the lattice [2,3]. It was derived by Ginsparg and Wilson in 1981 as the remnant of chiral symmetry on the lattice after blocking a chirally symmetric theory with a chirality breaking local renormalization group transformation. The GW relation for exactly massless Dirac fermion operator $D$ is

$D \gamma_{5}+\gamma_{5} D=2 D \gamma_{5} R D$

where the chiral symmetry breaking on the RHS of Eq. (1) constitutes an invertible hermitian operator $R$ which is local in the position space and trivial in the Dirac space.

In topologically trivial background gauge field, $D^{-1}$ exists and Eq. (1) can be written in terms of the fermion propagator $D^{-1}$

$D^{-1} \gamma_{5}+\gamma_{5} D^{-1}=2 \gamma_{5} R$

This equation displays explicitly that the chirality

\footnotetext{
${ }^{1}$ E-mail: twchiu@phys.ntu.edu.tw
}

breaking part in the $\mathrm{GW}$ fermion propagator is dictated by the local operator $R$, in contrast to those non-local breakings due to adding a chiral symmetry breaking term explicitly to the action.

The GW relation, Eq. (1) can be rewritten as

$D \gamma_{5}(\mathbb{1}-R D)+(\mathbb{1}-D R) \gamma_{5} D=0$

Then it becomes evident that the lattice action $A=$ $\bar{\psi} D \psi$ is invariant under the exact chiral transformation on the lattice [4]:

$$
\begin{aligned}
\psi & \rightarrow \exp \left[i \theta \gamma_{5}(\mathbb{1}-R D)\right] \psi \\
\bar{\psi} & \rightarrow \bar{\psi} \exp \left[i \theta(\mathbb{1}-D R) \gamma_{5}\right]
\end{aligned}
$$

where $\theta$ is a global parameter. Consequently the axial anomaly can be deduced from the change of fermion integration measure under the exact chiral transformation (4), (5), and its sum over all sites is equal to the index of $D$ which is a well defined integer [5,4]. The Ginsparg-Wilson fermion circumvents the Nielson-Ninomiya no-go theorem [6] by 
breaking the continuum chiral symmetry $\left\{\gamma_{5}, D\right\}=0$ but keeping the exact chiral symmetry on the lattice, Eq. (3), hence $D$ can be constructed to be local and free of species doubling.

The salient feature of the GW relation Eq. (1) in topologically nontrivial background gauge field is that its chiral symmetry breaking part does not have any effects on the zero modes. This ensures that $D$ has exact zero modes with definite chiralities. Consequently the axial anomaly and the index of $D$ are invariant with respect to $R$, and thus they are determined solely by the chirally symmetric operator $D_{c}$ in the chiral limit $R \rightarrow 0$, where the GW relation is completely turned off. The role of the GW relation is to transform the nonlocal and sometimes singular (i.e., divergent) $D_{c}$ into a class of local and well defined fermion operators $D$ while keeping the topological characteristics of $D_{c}$ invariant. If we have a $D_{c}$ which can possess nonzero indices, then any $D$ constructed from this $D_{c}$ using the general solution of GW relation has the same topological characteristics. Conversely, if we have a Ginsparg-Wilson $D$ which has proper indices in topologically nontrivial gauge fields, then there must exist a chirally symmetric $D_{c}$ which has the same topological characteristics of $D$, and we can use this $D_{c}$ to construct a class of GW fermion operators. The purpose of this paper is to discuss the $R$-invariance of the axial anomaly, the index and the Atiyah-Singer index theorem on the lattice, and show that their realizations on the lattice are actually beyond the control of the GW relation but determined solely by the topological characteristics of the chirally symmetric $D_{c}$ in the chiral limit $R \rightarrow 0$. For the axial anomaly recently constructed by Lüscher [7], we show that the term proportional to $F \tilde{F}$ is independent of $R$ but depends on the topological characteristics of $D_{c}$, while the divergence term may depend on $R$ in a very complicated way, but we show that its relationship to the divergence of axial vector current is $R$-invariant.

We begin by reviewing the general solution of the Ginsparg-Wilson relation which has been discussed in Ref. [8]. Our purpose here is to set up the notations. The general solution of Eq. (1) can be formally represented by

$D=D_{c}\left(\mathbb{1}+R D_{c}\right)^{-1}=\left(\mathbb{1}+D_{c} R\right)^{-1} D_{c}$ where $D_{c}$ is the chirally symmetric fermion operator of Eq. (1) in the chiral limit $R \rightarrow 0$,

$D_{c} \gamma_{5}+\gamma_{5} D_{c}=0$

It is instructive to show that these two different forms of the general solution in (6) are equal and they satisfy Eq. (1). The details are shown in the following.

$$
\begin{aligned}
D_{c} & \left(\mathbb{1}+R D_{c}\right)^{-1} \\
& =\left(\mathbb{1}+D_{c} R\right)^{-1}\left(\mathbb{I}+D_{c} R\right) D_{c}\left(\mathbb{1}+R D_{c}\right)^{-1} \\
& =\left(\mathbb{I}+D_{c} R\right)^{-1} D_{c}\left(\mathbb{I}+R D_{c}\right)\left(\mathbb{1}+R D_{c}\right)^{-1} \\
& =\left(\mathbb{1}+D_{c} R\right)^{-1} D_{c}
\end{aligned}
$$

Using the general solution (6) to substitute $D$ on the RHS of Eq. (1), and inserting Eq. (7), we obtain

$2 \mathrm{D} \gamma_{5} R D$

$$
\begin{aligned}
= & 2\left(\mathbb{1}+D_{c} R\right)^{-1} D_{c} \gamma_{5} R D_{c}\left(\mathbb{I}+R D_{c}\right)^{-1} \\
= & \left(\mathbb{1}+D_{c} R\right)^{-1} D_{c} R \gamma_{5} D_{c}\left(\mathbb{1}+R D_{c}\right)^{-1} \\
& +\left(\mathbb{1}+D_{c} R\right)^{-1} D_{c} \gamma_{5} R D_{c}\left(\mathbb{1}+R D_{c}\right)^{-1} \\
& +\left(\mathbb{I}+D_{c} R\right)^{-1}\left(\gamma_{5} D_{c}+D_{c} \gamma_{5}\right)\left(\mathbb{1}+R D_{c}\right)^{-1} \\
= & \left(\mathbb{1}+D_{c} R\right)^{-1}\left(\mathbb{1}+D_{c} R\right) \gamma_{5} D_{c}\left(\mathbb{1}+R D_{c}\right)^{-1} \\
& +\left(\mathbb{1}+D_{c} R\right)^{-1} D_{c} \gamma_{5}\left(\mathbb{1}+R D_{c}\right)\left(\mathbb{I}+R D_{c}\right)^{-1} \\
= & \gamma_{5} D+D \gamma_{5}
\end{aligned}
$$

For any $D_{c}$ (hence $D$ ) satisfying the hermiticity condition

$\gamma_{5} D_{c} \gamma_{5}=D_{c}^{\dagger}$

then $D_{c}$ is antihermitian and it is evident from [8] that there is one to one correspondence ${ }^{2}$ between $D_{c}$ and a unitary operator $V$ satisfying the hermiticity condition $\left(\gamma_{5} V \gamma_{5}=V^{\dagger}\right)$

$$
\begin{aligned}
& D_{c}=(\mathbb{I}+V)(\mathbb{I}-V)^{-1}, \\
& V=\left(D_{c}-\mathbb{1}\right)\left(D_{c}+\mathbb{1}\right)^{-1}
\end{aligned}
$$

It is interesting to note that $V$ exists for all $D_{c}$, in particular, when $D_{c} \rightarrow \pm i \infty, V \rightarrow+1$; but when

\footnotetext{
${ }^{2}$ This observation is made by Herbert Neuberger in a private communication to the author.
} 
$D_{c} \rightarrow 0, V \rightarrow-1$. The eigenvalues of $V$ fall on a unit circle with center at zero. On the other hand, $D_{c}$ becomes singular (with simple poles) when $V$ has real eigenvalues +1 . This is exactly what we want it to happen when the background gauge field is topologically nontrivial. However $D$ is still well defined even when $D_{c}$ is singular. Substituting $D_{c}$ into (6), we obtain the general solution of $D$ in terms of a unitary operator $V$ satisfying the hermiticity condition [8],

$$
\begin{aligned}
D & =(\mathbb{1}+V)[(\mathbb{1}-V)+R(\mathbb{1}+V)]^{-1} \\
& =[(\mathbb{1}-V)+(\mathbb{1}+V) R]^{-1}(\mathbb{1}+V)
\end{aligned}
$$

The general solution Eqs. (10) and (11) can be casted into many different forms, for example [3],

$D=\frac{1}{\sqrt{2 R}}(\mathbb{1}+W) \frac{1}{\sqrt{2 R}}$

where $W$ is a unitary operator which can be expressed in terms of the $V$ and $R$. However, since $W$ depends on $R$, using this form could easily obscure some simple facts especially in the chiral limit $R \rightarrow$ 0 . Therefore we refrain from using Eq. (12) for analytic studies involving the general solution of the GW relation.

In general, the chirally symmetric operator $D_{c}$ is constructed such that it agrees with the continuum Dirac operator $\gamma_{\mu}\left(\partial_{\mu}+i A_{\mu}\right)$ in the classical continuum limit. It is obvious from Eq. (6) that if $D_{c}$ has species doubling, $D$ must have species doubling too. In order to avoid species doubling for a chirally symmetric operator, $D_{c}$ must be nonlocal. We refer to Ref. [9] for a few explicit examples of $D_{c}$. Next we consider the topological characteristics of a Dirac operator $D$ by comparing its index, index $(D)$ to the topological charge $Q$ of the smooth background gauge field. The index of $D$ is defined as $N_{-}-N_{+}$ where $N_{+}\left(N_{-}\right)$denotes the number of zero modes of $D$ with positive (negative) chirality. If index $(D)$ $=0$ for any smooth gauge configurations, then $D$ is called topologically trivial. If index $(D)=Q$, then $D$ is called topologically proper. If $D$ is not topologically trivial but index $(D) \neq Q$, then $D$ is called topologically improper. Examples of these three different cases are given below and detailed discussions in Ref. [10]. From Eq. (6), it is obvious that any zeromode of $D$ is a zeromode of $D_{c}$ and vice versa.
Therefore the index of $D$ is the same as the index of $D_{c}$, independent of $R$.

$$
\begin{aligned}
\operatorname{index}[D, A] & =\operatorname{index}\left[D_{c}, A\right] \\
& =N_{-}\left[D_{c}, A\right]-N_{+}\left[D_{c}, A\right]
\end{aligned}
$$

where we have indicated explicitly that the number of zero modes and the index are functionals of $D_{c}$ (D) and the gauge field $A$. However, if $D_{c}$ is well defined, its index must be zero. This can be easily proved in the following.

Proof: Consider $R=r \mathbb{1}$, then $\operatorname{index}(D)=$ $r \sum_{x} \operatorname{tr}\left[\gamma_{5} D(x, x)\right][5,4]$. In the limit $r \rightarrow 0, D \rightarrow D_{c}$, if $D_{c}$ is well defined, then $\sum_{x} \operatorname{tr}\left[\gamma_{5} D(x, x)\right]$ is finite, thus multiplying $r$ gives $\operatorname{index}(D)=0$. This completes the proof.

Therefore in order for $D_{c}$ to possess non-zero indices, $D_{c}$ must be singular (or equivalently, $V$ has real eigenvalue pairs \pm 1 ) in topologically nontrivial background gauge field [8].

To summarize above discussions, we list the necessary requirements for $D_{c}$ to enter Eq. (6) for constructing a local and well defined $D$ such that $D$ could possibly reproduce the continuum physics:

(a) $D_{c}$ is chirally symmetric and agrees with $\gamma_{\mu}\left(\partial_{\mu}+\right.$ $i A_{\mu}$ ) in the classical continuum limit.

(b) $D_{c}$ is free of species doubling.

(c) $D_{c}$ is nonlocal.

(d) $D_{c}$ is well defined in topologically trivial background gauge field.

(e) $D_{c}$ is singular (or equivalently, $V$ has real eigenvalue pairs \pm 1 ) in topologically non-trivial background gauge field.

It should be noted that each one of these constraints is required to be satisfied. For example, if (b) is not satisfied, then the index of $D_{c}$ must be incorrect no matter other constraints are satisfied or not. The most difficult task is to find a $D_{c}$ satisfying (e).

At this point, it is instructive to consider examples of topologically proper, trivial and improper $D_{c}$ respectively. First we consider the Neuberger-Dirac operator [11] in the chiral limit $(R \rightarrow 0)$

$D_{c}=2 M \frac{\mathbb{1}+V}{\mathbb{1}-V}, V=D_{w}\left(D_{w}^{\dagger} D_{w}\right)^{-1 / 2}$

where $M$ is an arbitrary mass scale, $D_{w}$ is the Wilson-Dirac fermion operator with negative mass $-m_{0}$ and Wilson parameter $r_{w}>0$

$D_{w}=-m_{0}+\frac{1}{2}\left[\gamma_{\mu}\left(\nabla_{\mu}^{*}+\nabla_{\mu}\right)-r_{w} \nabla_{\mu}^{*} \nabla_{\mu}\right]$ 
where $\nabla_{\mu}$ and $\nabla_{\mu}{ }^{*}$ are the forward and backward difference operators defined in the following,

$$
\begin{aligned}
& \nabla_{\mu} \psi(x)=U_{\mu}(x) \psi(x+\hat{\mu})-\psi(x) \\
& \nabla_{\mu}^{*} \psi(x)=\psi(x)-U_{\mu}^{\dagger}(x-\hat{\mu}) \psi(x-\hat{\mu})
\end{aligned}
$$

Using Eq. (6), we obtain the generalized NeubergerDirac operator

$$
D=2 M(\mathbb{1}+V)[(\mathbb{1}-V)+2 M R(\mathbb{1}+V)]^{-1}
$$

For $m_{0} \in\left(0,2 r_{w}\right)$, both $D$ and $D_{c}$ are topologically proper and reproduce the exact index theorem on a finite lattice for smooth background gauge fields as first demonstrated in $[13,14]$. On the other hand, for $m_{0} \leq 0$, that is, $D_{w}$ with a positive mass, then $D$ and $D_{c}$ becomes topologically trivial [12]. We note that in both trivial and proper cases $D$ is free of species doubling and is local for properly chosen $R$. For $m_{0} \geq 2 r_{w}$, both $D$ and $D_{c}$ are either topologically improper or trivial. We emphasize that these ranges of $m_{0}$ values depend on the gauge configurations, and the ranges given above are exact only in the free field limit. We refer to Ref. [10] for further discussions on the topological characteristics of the Neuberger-Dirac operator.

It is interesting to note that the $G W$ relation does not play any role in determining the index of $D_{c}$. The GW relation only ensures that the chiral symmetry is broken in such a delicate way that the exact zero modes of $D_{c}$ are also exact zero modes of $D$ with the same chiralities, and thus the index of $D$ is equal to the index of $D_{c}$. The role of $R$ is to convert the nonlocal and sometimes singular $D_{c}$ into a local and well defined $D$, as well as to determine the locality of $D$. If one could not find any $D_{c}$ satisfying all constraints (a)-(e), then the GW relation does not have much use at all. On the other hand, if one knows one $D_{c}$ satisfying all constraints (a)-(e), then the resulting $D$ must have exact chiral symmetry on the lattice as well as all attractive features pointed out in $[15,16]$.

The fermion integration measure in general is not invariant under the exact chiral transformation (4), (5), and this gives the axial anomaly on the lattice [4]

$$
q(x, A ; D)=\operatorname{tr}\left[\gamma_{5}(R D)(x, x)\right]
$$

where tr denotes the trace over Dirac indices. We note that $q(x, A ; D)$ is a function of $x$ and the gauge field $A_{\mu}$ in the neighborhood around $x$, and a functional of $D$. The sum of $q(x, A ; D)$ over all lattice sites is shown $[5,4,11]$ to equal to the index of $D$

$$
\begin{aligned}
\sum_{x} q(x, A ; D) & =\sum_{x} \operatorname{tr}\left[\gamma_{5}(R D)(x, x)\right] \\
& =N_{-}[D, A]-N_{+}[D, A] \\
& =\operatorname{index}[D, A]
\end{aligned}
$$

where $N_{+}\left(N_{-}\right)$denotes the number of zero modes of $D$ with positive (negative) chirality. In this paper, we restrict our discussions to $U(1)$ gauge theory with single fermion flavor. Since the index of $D$ is independent of $R$ [Eq. (13)], the total axial anomaly must be invariant under any smooth deformations of $R$

$\delta_{R} \sum_{x} q(x, A ; D)=0$

Recently Lüscher [7] has proved that for $R=1 / 2$, if the axial anomaly $q(x, A ; D)$ satisfies

$\sum_{x} \delta q(x, A ; D)=0$

for any local deformations of the gauge field, then

$$
\begin{aligned}
q(x, A ; D)= & \gamma c[D] \epsilon_{\mu \nu \lambda \sigma} F_{\mu \nu}(x) F_{\lambda \sigma}(x+\hat{\mu}+\hat{\nu}) \\
& +\partial_{\mu} G_{\mu}(x, A ; D)
\end{aligned}
$$

where $\gamma$ is a constant, $c[D]$ is a functional of $D$ which only depends on the topological characteristics of $D$, and

$$
\begin{aligned}
& \partial_{\mu} G_{\mu}(x, A ; D) \\
& \quad=\sum_{\mu}\left[G_{\mu}(x, A ; D)-G_{\mu}(x-\hat{\mu}, A ; D)\right]
\end{aligned}
$$

The explicit form of the current $G_{\mu}(x, A ; D)$ is supposed to be very complicated. We note that $c[D]$ is not shown explicitly in [7]. Since the total axial anomaly is invariant under smooth deformations of $R$, Eq. (19), as well as for any local deformations of the gauge field, Eq. (20), then the theorem proved by Lüscher [7] can be applied to the case of the general $R^{3}$, and the axial anomaly can be written in the same form as Eq. (21) on an infinite lattice. But on a

\footnotetext{
${ }^{3}$ This is confirmed by Martin Lüscher in a private communication to the author.
} 
finite lattice, $q(x, A ; D)$ must be modified by the boundary effects. However, if $D$ is local, the boundary effects are negligible, then Eq. (21) can be used for the axial anomaly on a finite lattice. Since the locality of $D$ is controlled by $R$, we can infer that all boundary effects must have dependence on $R$ and hence they do not enter the $F \tilde{F}$ term. [As we will show later, the $F \tilde{F}$ term does not depend on $R$.] Furthermore they must be in the form of the divergence term, otherwise Eq. (21) cannot be consistent with Eq. (28), the divergence of axial vector current on a finite lattice. Therefore we conclude that Eq. (21) is applicable for a finite lattice as well as for an infinite lattice, and the boundary effects only enter the divergence term. Now we proceed our discussions for a finite lattice embedded in a 4-dimensional torus. After summing $q(x, A ; D)$ over all sites, the divergence term is removed, then Eq. (18) gives

$$
\begin{aligned}
\gamma c & {[D] \sum_{x} \epsilon_{\mu \nu \lambda \sigma} F_{\mu \nu}(x) F_{\lambda \sigma}(x+\hat{\mu}+\hat{\nu}) } \\
& =N_{-}[D, A]-N_{+}[D, A]=\operatorname{index}[D, A]
\end{aligned}
$$

Since index $[D, A]$ is independent of $R$ [Eq. (13)], then Eq. (23) implies that $c[D]$ must be also independent of $R$,

$c[D]=c\left[D_{c}\right]$

So we have proved that all $R$ dependence in $q(x, A ; D)$ only resides in the current $G_{\mu}(x, A ; D)$. Then Eq. (23) can be rewritten in the following.

$$
\begin{aligned}
\gamma c & {\left[D_{c}\right] \sum_{x} \epsilon_{\mu \nu \lambda \sigma} F_{\mu \nu}(x) F_{\lambda \sigma}(x+\hat{\mu}+\hat{\nu}) } \\
& =N_{-}\left[D_{c}, A\right]-N_{+}\left[D_{c}, A\right]=\operatorname{index}\left[D_{c}, A\right]
\end{aligned}
$$

Since index $\left[D_{c}, A\right]=0$ if $D_{c}$ is topologically trivial, then $c\left[D_{c}\right]$ also vanishes for trivial $D_{c}$. Now we consider two topologically proper $D_{c}$ which are identical except their electric charges of the fermion field. Then for a given topologically non-trivial gauge configuration, Eq. (25) implies that the ratio of $c\left[D_{c}\right]^{\prime} s$ corresponding to these two $D_{c}$ must equal to the ratio of two integers corresponding to their indices. It follows from this that $c\left[D_{c}\right]$ must be an integer. Therefore we can fix $c\left[D_{c}\right]=1$ for electric charge equal to one and then determine the value of the constant $\gamma$ by evaluating $\sum_{x} \epsilon_{\mu \nu \lambda \sigma} F_{\mu \nu}(x) F_{\lambda \sigma}(x$ $+\hat{\mu}+\hat{\nu})$ on the LHS for the simplest nontrivial gauge configuration with constant field tensor, namely, $F_{12}=2 \pi /\left(L_{1} L_{2}\right)$ and $F_{34}=2 \pi /\left(L_{3} L_{4}\right)$ while other $F^{\prime} s$ are zero. After restoring the unit of electric charge, we obtain $\gamma$ to be integer multiple of $e^{2} /\left(32 \pi^{2}\right)$ for $U(1)$ gauge theory with single fermion flavor. For Neuberger-Dirac fermion operator [11], this integer is determined to be unity by a recent perturbation calculation of $q(x, A ; D)$ [17]. For the general Ginsparg-Wilson fermion with $R=r \mathbb{1}$, and $D_{c}$ satisfying constraints (a)-(e) ${ }^{4}$, our recent perturbation calculation [18] of $q(x, A ; D)$ show that $\gamma$ is independent of $r$ and equal to $e^{2} /\left(32 \pi^{2}\right)$. Then Eq. (25) becomes

$$
\begin{aligned}
& \frac{e^{2}}{32 \pi^{2}} c\left[D_{c}\right] \sum_{x} \epsilon_{\mu \nu \lambda \sigma} F_{\mu \nu}(x) F_{\lambda \sigma}(x+\hat{\mu}+\hat{\nu}) \\
& \quad=N_{-}\left[D_{c}, A\right]-N_{+}\left[D_{c}, A\right]
\end{aligned}
$$

where

$$
\begin{aligned}
& c\left[D_{c}\right] \\
& = \begin{cases}1 & \text { if } D_{c} \text { is topologically proper } \\
0 & \text { if } D_{c} \text { is topologically trivial } \\
\text { integer } \neq 0,1 & \text { if } D_{c} \text { is topologically improper }\end{cases}
\end{aligned}
$$

For any chirally symmetric $D_{c}$ satisfying constraints (a)-(e), Eq. (26) is the exact realization of the Atiyah-Singer index theorem on the lattice and it holds for any Ginsparg-Wilson fermion operator $D=D_{c}\left(\mathbb{1}+R D_{c}\right)^{-1}$.

Although the explicit form of the current $G_{\mu}(x, A ; D)$ in Eq. (21) is supposed to be very complicated, however, it is instructive to compare Eq. (21) to the divergence of axial vector current on the lattice $[1,5]$,

$$
\begin{aligned}
\partial_{\mu}\left\langle J_{\mu}^{5}(x, A ; D)\right\rangle & \\
= & 2 q(x, A ; D)+2 \sum_{s}^{N_{+}}\left[\phi_{+}^{s}(x)\right]^{\dagger} \phi_{+}^{s}(x) \\
& -2 \sum_{t}^{N_{-}}\left[\phi_{-}^{t}(x)\right]^{\dagger} \phi_{-}^{t}(x)
\end{aligned}
$$

where $\phi_{+}^{s}$ and $\phi_{-}^{t}$ are normalized eigenfunctions of

\footnotetext{
${ }^{4}$ In Ref. [18], it is only required that the chiral fermion operator $D_{c}(p)$ is free of species doubling and in the free field limit behaves like $i \gamma_{\mu} p_{\mu}$ as $p \rightarrow 0$.
} 
$D\left(D_{c}\right)$ with eigenvalues $\lambda_{s}=\lambda_{t}=0$ and chiralities +1 and -1 respectively. Eliminating $q(x, A ; D)$ from both equations, we obtain

$$
\begin{aligned}
\partial_{\mu} G_{\mu}(x, A ; D) & \\
= & \frac{1}{2} \partial_{\mu}\left\langle J_{\mu}^{5}(x, A ; D)\right\rangle \\
& -\frac{e^{2}}{32 \pi^{2}} c[D] \epsilon_{\mu \nu \lambda \sigma} F_{\mu \nu}(x) F_{\lambda \sigma}(x+\hat{\mu}+\hat{\nu}) \\
& -\sum_{s}^{N_{+}}\left[\phi_{+}^{s}(x)\right]^{\dagger} \phi_{+}^{s}(x) \\
& +\sum_{t}^{N_{-}}\left[\phi_{-}^{t}(x)\right]^{\dagger} \phi_{-}^{t}(x)
\end{aligned}
$$

It is interesting to note that all dependences on $R$ are in the currents $G_{\mu}$ and $J_{\mu}^{5}$. The zero modes and the $F \tilde{F}$ term do not depend on $R$ as we have discussed above. Then we obtain

$\delta_{R} \partial_{\mu}\left[G_{\mu}(x, A ; D)-\frac{1}{2}\left\langle J_{\mu}^{5}(x, A ; D)\right\rangle\right]=0$

This equation provides a $R$-invariant constraint between the current $G_{\mu}(x, A ; D)$ and the axial vector current $J_{\mu}^{5}(x, A ; D)$. The axial vector current $J_{\mu}^{5}(x, A ; D)$ is defined [1] in terms of the kernel $K_{\mu}^{5}(x, y, z, A ; D)$ through the equation

$J_{\mu}^{5}(x, A ; D)=\sum_{y, z} \bar{\psi}_{x+y} K_{\mu}^{5}(x, y, z, A ; D) \psi_{x+z}$

where $K_{\mu}^{5}$ is expressed in terms of the kernel $K_{\mu}$ for the vector current,

$K_{\mu}^{5}=\frac{1}{2}\left(K_{\mu} \gamma_{5}-\gamma_{5} K_{\mu}\right)$

Ginsparg and Wilson [1] proved that the kernel for the vector current $K_{\mu}(x, y, z, A ; D)$ is equal to $D(x$ $+y, x+z, A)$ times the sign of $(y-z)_{\mu}$ and times the fraction of the shortest length paths from $x+z$ to $x+y$ which pass through the link from $x-\hat{\mu}$ to $x$. Finally the axial anomaly in Eq. (21) becomes

$$
\begin{aligned}
q(x, A ; D)= & \frac{e^{2}}{32 \pi^{2}} c\left[D_{c}\right] \epsilon_{\mu \nu \lambda \sigma} F_{\mu \nu}(x) F_{\lambda \sigma} \\
& \times(x+\hat{\mu}+\hat{\nu})+\partial_{\mu} G_{\mu}(x, A ; D)
\end{aligned}
$$

where $c\left[D_{c}\right]$ is defined in Eq. (27) and the divergence term $\partial_{\mu} G_{\mu}(x, A ; D)$ is related to the divergence of axial vector current by Eqs. (29) and (30).
To summarize, we have clarified the role of the Ginsparg-Wilson relation in the realization of the axial anomaly and the exact Atiyah-Singer index theorem on the lattice. The crucial point is the existence of a topologically proper chirally symmetric Dirac operator $D_{c}$ in the chiral limit where the Ginsparg-Wilson relation is turned off. If $D_{c}$ satisfies the constraints (a)-(e) discussed in this paper, then any Ginsparg-Wilson fermion operator $D$ constructed by Eq. (6) could reproduce the correct continuum physics, in particular, the correct axial anomaly and the exact index theorem. From this point of view, the underlying reason why Neuberger-Dirac fermion really works is because the existence of the topologically proper $D_{c}$ (or $V$ ) [Eq. (14)] which stems from the Overlap formalism [19,2]. The Ginsparg-Wilson relation only provides a scheme to break the continuum chiral symmetry in such a delicate way that the exact chiral symmetry on the lattice is preserved and the axial anomaly and the index are invariant while smooth deformations of $R$ away from zero bring the non-local and sometimes singular $D_{c}$ into a class of local and well defined Ginsparg-Wilson fermion operators $D$. The most crucial and highly non-trivial task is to find a 'good seed' $D_{c}$ which at least satisfies all constraints (a) (e). So far there is only one good $D_{c}$ found explicitly. However, there is no indications that $D_{c}$ is unique and thus the search for computationally less expensive $D_{c}$ is highly desirable. Since the Overlap formalism did provide vital insights in discovering a good $D_{c}$ [11], it would be interesting to see whether it could guide us to find a better one. Mathematically, it is interesting to understand what actually determines the topological characteristics of an operator and how to construct a topologically proper operator in a systematic way. For the axial anomaly (21) recently constructed by Lüscher [7], we show that the $F \tilde{F}$ term is $R$-invariant and the proportional constant is $e^{2} /\left(32 \pi^{2}\right)$ times $c\left[D_{c}\right]$ which only depends on the topological characteristics of $D_{c}$; and the divergence term is related to the divergence of axial vector current by an $R$-invariant constraint, Eqs. (29) and (30). For any chirally symmetric $D_{c}$ satisfying constraints (a)-(e), the Atiyah-Singer index theorem can be realized exactly on the lattice, Eq. (26), and it holds for any Ginsparg-Wilson fermion operator $D=D_{c}\left(\mathbb{1}+R D_{c}\right)^{-1}$. 


\section{Acknowledgements}

I would like to thank Martin Lüscher and Herbert Neuberger for enlightening correspondences. I also wish to thank Sergei V. Zenkin for many discussions in the last few months. This work was supported by the National Science Council, ROC under the grant number NSC88-2112-M002-016.

\section{References}

[1] P. Ginsparg, K. Wilson, Phys. Rev. D 25 (1982) 2649.

[2] For a recent review and references, see H. Neuberger, Chirality on the lattice, hep-lat/9808036.

[3] F. Niedermayer, plenary talk at Lattice 98, Boulder, July 13-18, 1998.

[4] M. Lüscher, Phys. Lett. B 428 (1998) 342.

[5] P. Hasenfratz, V. Laliena, F. Niedermayer, Phys. Lett. B 427 (1998) 125
[6] H.B. Nielsen, N. Ninomiya, Nucl. Phys. B 185 (1981) 20; B 193 (1981) 173.

[7] M. Lüscher, Topology and the axial anomaly in abelian lattice gauge theories, hep-lat/9808021.

[8] T.W. Chiu, S.V. Zenkin, On solutions of the Ginsparg-Wilson relation, hep-lat/9806019.

[9] T.W. Chiu, C.W. Wang, S.V. Zenkin, Phys. Lett. B 438 (1998) 321.

[10] T.W. Chiu, Topological phases of Neuberger-Dirac operator, hep-lat/9810002.

[11] H. Neuberger, Phys. Lett. B 417 (1998) 141.

[12] H. Neuberger, Phys. Lett. B 427 (1998) 353.

[13] T.W. Chiu, Phys. Rev. D 58 (1998) 074511.

[14] T.W. Chiu, Exactly massless fermions on the lattice, heplat/9809085.

[15] P. Hasenfratz, Nucl. Phys. B 525 (1998) 401.

[16] S. Chandrasekharan, Lattice QCD with Ginsparg-Wilson fermions, hep-lat/9805015.

[17] Y. Kikukawa, A. Yamada, Weak coupling expansion of massless QCD with a Ginsparg-Wilson fermion and axial U(1) anomaly, hep-lat/9806013.

[18] T.W. Chiu, T.H. Hsieh, Perturbation calculation of axial anomaly of Ginsparg-Wilson fermion, NTUTH-98-098.

[19] R. Narayanan, H. Neuberger, Nucl. Phys. B 443 (1995) 305. 